\title{
Nuevos paseantes. El síntoma lacaniano aplicado al nomadismo inocente como producción cultural del "partener"
}

\author{
Aldo Enrici *, Luciana Mansilla **
}

\section{Resumen}

El 15 de marzo de 2021 un vagabundo llevó a pasear en bicicleta por la ciudad de Buenos Aires a una niña "en situación de calle". Recorrieron la ciudad relajados entre peatones y automóviles. Finalmente, fueron localizados. A pesar de la búsqueda de los medios, las redes sociales y el alerta policial, no se advirtió su presencia de estos paseantes que cruzaron la ciudad a la vista de todos.

El presente artículo procura vincular este episodio con el Seminario XXIII de Jacques Lacan sobre "el síntoma". Lacan, a partir del caso de James Joyce sostiene que desde el arte y el ingenio se desarrollan lazos originales cuando se disipan las reglas simbólicas.

Aún perdura la sospecha de criminalidad en los vagabundos. El vagabundo se asemeja a los nómades prehistóricos. A su vez una niña brutalizada por su falta hogar y de educación, es un caso de cuidado. Tanta carencia la vuelve un objeto inocente, una "buen salvaje". Consideraremos la teoría de Eric Laurent sobre el síntoma como partener de vida. El partener acompaña en el nomadismo como una forma de contacto para efectividad del lazo.

El paseo por la ciudad había sido considerado ya, por Charles Baudelaire, como una costumbre ciudadana. Una necesidad de convivencia con la muchedumbre. Consideraremos la vida nómade como una forma de escape de la persecución ciudadana, de la crisis laboral, de los costos de vivir en una vivienda. Se ponen en juego lazos creativos que hacen coincidir fragmentos de la personalidad. Los paseantes forman un síntoma cultural para evitar la identificación.

Palabras clave: $\quad$ Síntoma lacaniano - paseantes - nomadismo - producción cultural - partner Laurent 


\title{
The walkers. The Lacanian symptom applied to innocent nomadism as a cultural production of "partner" in civic conditions
}

\author{
Aldo Enrici *, Luciana Mansilla **
}

\begin{abstract}
On March 15, 2021, a homeless person took a girl "in a street situation" on a bicycle ride through the city of Buenos Aires. They walked through the city relaxed among pedestrians and cars. Finally, they were located. Despite the search by the media, social networks and the police alert, their presence was not noticed by these walkers who crossed the city in full view of all.

This article attempts to link this episode with Jacques Lacan's Seminar XXIII on "the symptom". Lacan, based on the case of James Joyce, argues that original ties are developed from art and ingenuity when symbolic rules dissipate.

The suspicion of criminality in the homeless still persists. The Wanderer resembles prehistoric nomads. At the same time, a girl brutalized by her lack of home and education, is a case of care. So much lack makes her an innocent object, a "good savage." We will consider Eric Laurent's theory of the symptom as a life partner. The partener accompanies nomadism as a form of contact for the effectiveness of the bond. The walk through the city had already been considered, by Charles Baudelaire, as a citizen custom. A need to coexist with the crowd. We will consider the nomadic life as a way of escape from citizen persecution, from the labor crisis, from the costs of living in a house. Creative ties are brought into play that bring fragments of the personality together. Walkers form a cultural symptom to avoid identification.
\end{abstract}

Keywords: Lacanian symptom - walkers - nomadism - cultural production partner Laurent 


\section{Introducción}

\section{El paseante (flâneur) como síntoma. De Baudelaire a Lacan}

Para Charles Baudelaire la multitud es como el aire para los pájaros y el agua para los peces. Su pasión y su profesión le llevan a hacerse una sola carne con la muchedumbre. Para el paseante (flâneur), es un júbilo establecer su morada en el corazón de la calle, entre el flujo y reflujo del movimiento, entre lo fugitivo y lo infinito. Charles Baudelaire, "El pintor de la vida moderna", (Nueva York: Da Capo Press, 1964). Orig. Publicado en Le Figaro (1863).

Los paseantes eran, ante todo, un tipo literario en la Francia del s. XIX. Charles Baudelaire los descubre. Eran la ilustración de las calles de París. Posteriormente, de toda gran ciudad. El explorador urbano, el individuo curtido en la calle, etcétera. Gregory Shaya (2004). Patologizar los desórdenes, los errores, las ilusiones de la locura, para lograr la limpieza pública, lleva a considerar que la elección de vida indigente es un síntoma de afección mental.

La propuesta psicoanalítica plantea el "síntoma" ( $\sigma u ́ \mu \pi \tau \omega \mu \alpha$ en griego) sirve de lazo que permite mantener el contacto de lo simbólico con lo real. Lacan le da valor al lazo sintomático como la des-simbolización por vía de la producción cultural. Da cuenta de "saber arreglárselas" con su síntoma.

Sostiene que el síntoma genera una producción artística desde la distorsión psíquica. Al borde de la psicosis se permite el brote de nuevas sensaciones estéticas. Esas sensaciones se vuelven paradigmáticas para una nueva literatura. Joyce no es una excepción, es un estándar. El síntoma compensa la des-simbolización. Se vuelve una "producción cultural" -pensemos en tres casos: Francis Bacon, Van Gogh, Frida Kahlo- . Nos referimos a productores de cultura, de arte, cuyas vidas demuestran dificultades de convivencia a partir de accidentes, delirios persecutorios, elecciones de género, reprobaciones sociales. Sin embargo, sus generaciones artísticas revolucionan las apreciaciones. No se autolimitan con los rigores de la racionalidad de su contexto.

\section{El nomadismo inocente de Joyce y la producción cultural}

El seminario a partir de la teoría del síntoma tal como lo expresa Jacques Lacan debe ser interpretado como una propuesta psicoanálisis que puede realizar aportes etnográficos. Sobre todo, hablamos de aportes para comprender aquellas figuraciones culturales que intentan mostrarnos una pequeña desviación o un modo de expresarse que pueda tener un alcance estético distinto y que está en el filo de una incipiente desimbolización. En el caso de Lacan su trabajo con Joyce es muy particular.

Joyce es un migrante. Es alguien que se va de su país natal, Irlanda. Atraviesa por fases de alcoholismo vinculado a la pobreza, a la pérdida de la vista, a viajes intelectuales por Europa sin un destino final. No tiene un destino fijo. Joyce se ampara en "la luz de sus 
ojos" que va a ser su hija Lucía. Lucía es precisamente el nombre, que concuerda con su déficit de la visión. La trayectoria de Lucía lo convoca a no derrumbarse en un abismo de psicosis. Joyce cree en ese partener. Cree a pesar de que era una niña que tuvo dificultades mentales y llegó a la locura. Así, la "visión" se le impuso a la hija de Joyce desde el nacimiento y, en el auge de su locura, esa imposición le retornará a Joyce como padre, vehiculizada nuevamente, a través de la atribución que él, tomando en serio lo que ella decía, comenzó a hacerle de una videncia. Lucia, en italiano, se traduce también como luz. Es notable que la locura de Lucía se vuelve manifiesta. Lucía se agrava en el mismo período en que la salud de los ojos de Joyce empeora considerablemente.

Lucía es alguien de extrema clarividencia para Joyce. Es su modo de hacer síntoma. Lucía le puede aportar (a él) una suerte de ancla para su desvarío. Es la partener que le permite hacer arte. La progresiva gravedad de la locura de su hija Lucía. El escritor prefería atribuir tal perturbación a la vida nómade y a la variedad de lenguas que él mismo impuso a sus familiares, por haber elegido el exilio y su íntegra dedicación a la literatura.

Joyce elegía decir que su hija era, en verdad, portadora de una increíble clarividencia (Hutchins, 1957, p. 169). No se trata de reconocer un síntoma exclusivo de ese escritor porque la imposición de la palabra es, conforme el mismo psicoanálisis nos enseña, un desdoblamiento de la dimensión parasitaria propia de la palabra que, como tal, afecta a todo ser humano.

Se considera decisivo destacar un dato que refuerza esa prolongación que se realiza entre la "clarividencia" atribuida a su pequeña Lucía (quien es internada por un delirio psicótico) y las palabras impuestas a Joyce. Lucía es su partener. Acompaña su viaje, le da luz a su creciente pérdida de estabilidad mental. Como atreve a dictaminar Sérgio Laia (2003, p. 3), Lucía, como primer nombre, permite al escritor asociar su elección a la Santa patrona de la visión. La locura de Lucía se vuelve manifiesta. Se agrava en el mismo período en que la salud de los ojos de Joyce empeora considerablemente. Se encamina, cada vez más, por aquel "libro de la noche" titulado Finnegans Wake. Joyce sigue a Lucía en sus alucinaciones líricas. Lucía salva a Joyce. Como si fuera una patrona de la escritura.

\section{La noticia. Ha desaparecido una niña}

El lunes 15 de marzo de 2021 se anunció la búsqueda de una niña de 7 años, desaparecida. La niña vive en situación de calle en el barrio porteño de Villa Lugano. Es intensamente buscada desde el lunes por su familia y por las fuerzas de seguridad. Su mamá denunció que un hombre se la llevó con la promesa de cambiar su bicicleta y nunca regresó (Cannataro, 2021). Según la denuncia, el lunes a las 11 de la mañana la madre de Maia le permitió a la nena que vaya con el hombre, identificado como Carlos S. a buscar otra bicicleta. Este hombre paraba en la misma zona que Maia y su mamá desde hacía tres semanas. 
Para que se conozca y se difunda el caso, un grupo de vecinos del Barrio Cildañez cortó la autopista Dellepiane, a la altura de Escalada, donde continúa el reclamo y el pedido de aparición de la niña. Se emitió la "Alerta Sofía", un programa de emergencia ante casos de niños, niñas o adolescentes desaparecidos que puedan encontrarse en grave peligro. El sistema de alerta difunde de manera inmediata la imagen e información del niño, niña o adolescente desaparecido a través de dispositivos móviles, medios de comunicación masiva como la televisión, el correo electrónico y las redes sociales.

En la mañana del miércoles se pudo ver en las cámaras del ramal Sarmiento (del martes por la mañana) al hombre con la niña y una bicicleta. Primero en la estación de Liniers y luego descendiendo en la estación de Castelar. La red de las cámaras urbanas de seguridad los mostró cruzando las calles entre los autos y los peatones. Los reporteros de televisión, las redes sociales, especularon que el hombre era psicótico y perverso. Ambos aparecieron en Luján el miércoles, aun con la bicicleta, caminando por la acera.

\section{El encuentro con lo inocente}

El encuentro con lo inocente (Maia) se asemeja a toparse con un arbusto salvaje en un jardín simétrico. Implica dar con un modo de ser no socializado, pero inocente. Es lo que un naturalista desearía encontrar como testimonio de su narración sobre la ternura de lo primitivo. Si un hombre mayor indigente genera miedo, una niña indigente provoca lo contrario. Ese contraste dominaba en las entrañas del pensamiento evolucionista. La educación era la encargada de transformar la inocencia en ciudadanía.

El historiador Arnoldo Canclini publica recientemente una novela denominada EI Fueguino. Se permite recrear, allí, ese mundo de los niños primitivos, basado en las memorias del archivo del marino Robert Fitz Roy, capitán del famoso Beagle. El viaje del barco H.M.S. Beagle se inició en diciembre de 1831 en el dique naval de Plymouth (Inglaterra). Se terminó en octubre de 1836 en el puerto de Falmouth. Tenía dos misiones: continuar con los trabajos cartográficos de la costa suramericana, y conseguir una determinación más precisa de la longitud terrestre (Nichols, 2003).

La novela ficcionaliza una versión del encuentro con los yaganes, los nativos originarios y nómades de Tierra del Fuego. Vale recordar que ese convoy llevaba entre sus tripulantes a Charles Darwin. La idea de evolución latía en la embarcación. El capitán decidió confinar a cuatro Yaganes para llevarlos a la Corona. La posibilidad de instruirlos y exponerlos en ferias era una empresa algo morbosa. El comportamiento de una niña muestra su inocencia aun mezclada con la naturaleza.

"se la notaba deseosa de divertirse aprendiendo palabras en inglés y, de ese modo, se había comenzado un inicio de intercambio...Uno de los médicos de a bordo, la peinó, ocupándose al mismo tiempo de reducir la abundante fauna que se mezclaba en su arisca cabellera." (Canclini, 2009, pág. 18) 
Jacques Lacan, en el Seminario XXIII entiende que se elabora desde el arte un nuevo vínculo cuando se pierden reglas simbólicas. Maia continúa el síntoma de su colega que la invita a pasear. Precisamente al no circular en automóvil, ni tener un móvil electrónico esfuma su rastro simbólico como lo hace Lucía, la hija de Joyce. El paseo por la ciudad sin ningún dispositivo digital es un estado de ánimo diferente y nuevo en el mundo. El "sujeto-prótesis" está intercomunicado en red mediante alguna prótesis de inteligencia artificial, generalmente un smartphone. En el caso de Maia estamos ante la presencia de un individuo des-simbolizado. Si un vagabundo y una niña recorren la ciudad en bicicleta y sin teléfono inteligente están de-simbolizados. Puesto que se vestían como gente en "situación de calle", con vestuario viejo y sucio. Nadie quiso sentar la vista en ellos. Nadie había visto la bicicleta en la que viajaban. No se percataron de su presencia.

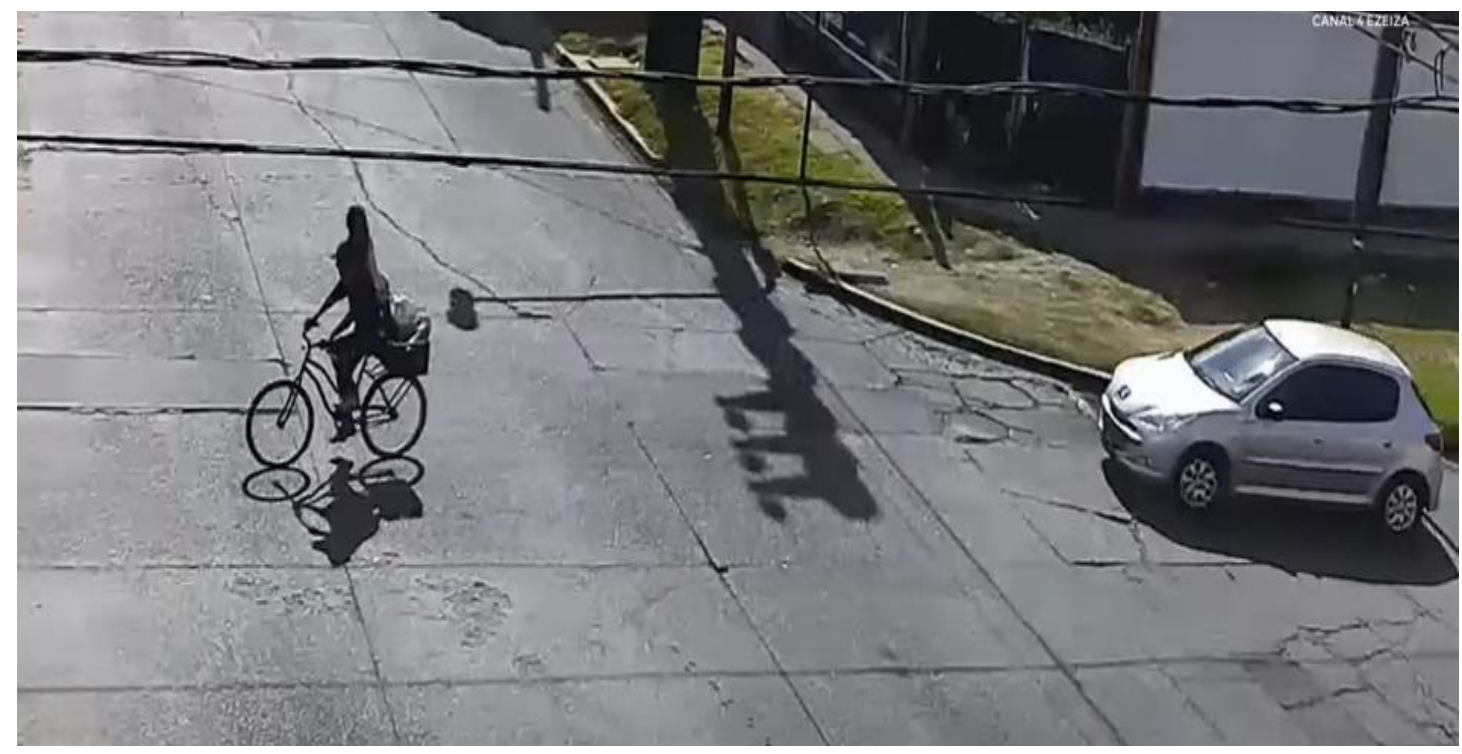

Maia en bicicleta, cruzando la ciudad.

https://www.elobservador.com.uy/nota/-donde-esta-maia-las-imagenes-de-la-menor-desiete-anos-y-su-secuestrador-que-impactan-buenos-aires-2021317151541

\section{La salud mental como higiene. Del criminal al objeto inocente}

Es una rutina periodística manejarse con la teoría sobre la "apariencia asesina" de los nómadas. Esta idea proviene de Cesare Lombroso (Gillebaud, 331). Lombroso asevera que el asesino es como un hombre primitivo. El criminal nato es una supervivencia asombrosa del salvaje primitivo. El hombre primitivo, según este punto de vista, se supone brutal por su falta de higiene, por su abandono a los placeres biológicos ilimitados. Satisface los instintos sin mediación sublime. O pertenece a un modo de vida primitivo, asociado a la ferocidad del resto de los animales, o padece una patología mental.

De esta conjetura se deriva una proximidad probable de criminalidad en los vagabundos. El vagabundo, "supuesto malviviente" con su pelo sucio, su hedor, o su falta de higiene, si seguimos esta presunción, no tiene hogar, ni trabajo, ni familia. Es un 
carroñero, similar a los nómadas prehistóricos. Por otro lado, una niña brutalizada por su falta de aseo, su falta de educación, es un caso de cuidado. Tanta carencia la vuelve un objeto inocente, una "buen salvaje".

La psiquiatría a principios del siglo XIX, advierte Michel Foucault (1991) como rama especializada de la higiene pública, como la profesión que lidera la higiene del cuerpo social en su totalidad. Se codifica la locura como enfermedad. La falta de higiene en el cuerpo es atribuible a la falta de adaptación social. Se patologiza el desorden. Fue necesario codificar la locura como peligro, portadora de riesgos. De este modo la psiquiatría, como saber de la enfermedad mental podía funcionar como la higiene pública.

La conciencia moderna europea otorga a la distinción entre lo normal y lo patológico el poder de delimitar lo irregular, lo desviado, lo poco razonable, lo criminal. Todo lo que se considera extraño recibe, en virtud de esta conciencia, el estatuto de la exclusión cuando se trata de juzgar y de la inclusión cuando se trata de explicar. Lo extraño oficia como la memoria interior cuando se rebela ante la educación. Michel Foucault (1991, p.7) mantiene que el mundo occidental conoció, y ello durante milenios, una medicina que se basaba en una conciencia de la enfermedad en la que lo normal y lo patológico no constituían los conceptos organizadores de las categorías fundamentales

El conjunto de las dicotomías fundamentales que, en nuestra cultura, se distribuyen a ambos lados del límite, entre las conformidades y las desviaciones, encuentra una justificación. Hablamos de la psiquiatría. Hizo funcionar la higiene pública como medicina y prevención, como curación eventual de la enfermedad mental. Actuaba como precaución social. Una vigilancia epistémica del paseo ciudadano. (Foucault, (19741975, pp. 335-339). A la psiquiatría se la consideró necesaria si se querían evitar cierta cantidad de peligros fundamentales. Peligros ligados a la existencia misma de la locura.

\section{El "síntoma" como partener de vida}

Lo saludable puede ser una invitación a la poesía, una invitación a hablar. Es decirle "no" a describir, "no" a explicar y verdaderamente "no" a decir la verdad. Una invitación pura y simplemente a hablar. La obra de arte hace posible el encuentro con lo real en tanto registro psíquico. No es imaginable ni se puede simbolizar, pero que se vincula a ellos. A partir del estudio de la obra de James Joyce, Jacques Lacan postula la idea de un "saber-hacer" específico del artista con el síntoma.

El "síntoma" mantiene precariamente unidos lo real y lo imaginario, por un lado, con un deficitario registro de lo simbólico por el otro. La existencia del "síntoma" permite a personalidades con derrumbes psicóticos mantener aspectos de personalidad normal (Lacan, 2013, p. 248). Eric Laurent (2019), entiende que el síntoma configura una época. La época de "aceptación del síntoma de cada uno, uno por uno".

Toda clasificación se vuelve inestable. El síntoma se ha transformado en un modo de frenar la noción agresiva de higiene pública como criterio para controlar al ciudadano que nos pone en peligro. Al reprimir las pulsiones no se hace más que acentuar el 
imperativo de superyó con su forma insaciable. Frente a esta creencia de un goce sin fin, emerge la creencia en el síntoma como partener. El partener-síntoma, como medio de goce, da forma y punto de detención a la búsqueda superyoica del gozar.

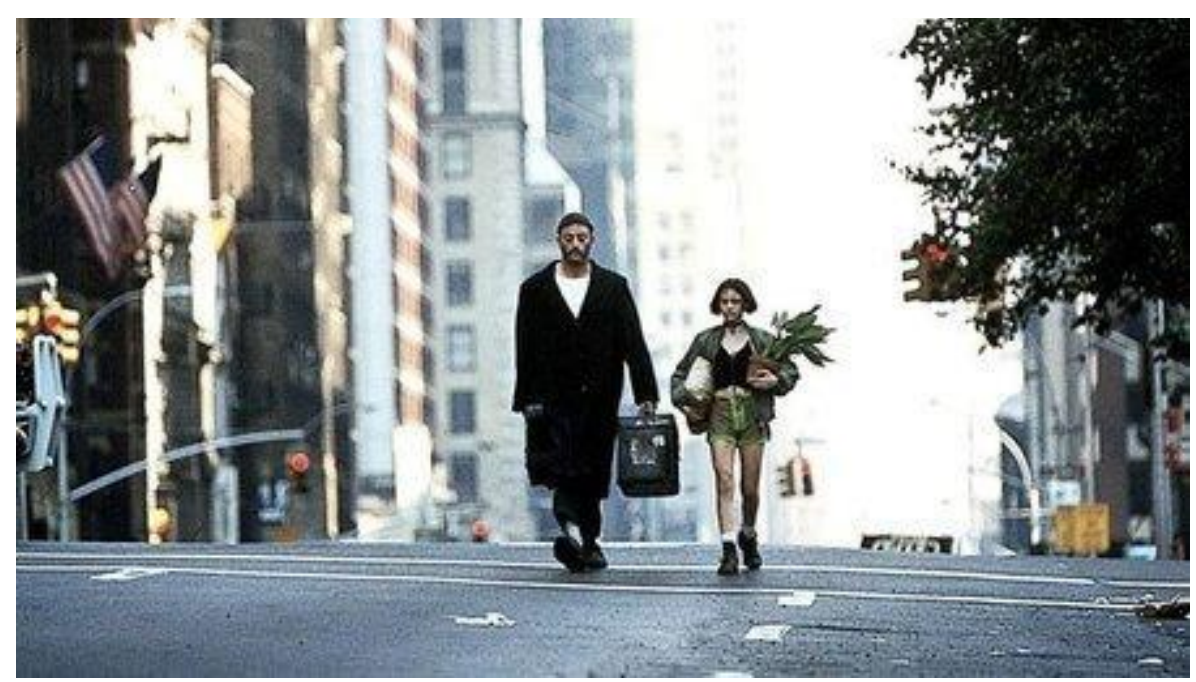

León y Matilda. En el filme "Leon el Profesional".

https://www.espinof.com/criticas/criticas-a-la-carta-el-profesional-leon

El síntoma como partener conlleva una complicidad inesperada. Alguien se constituye en partener cuando aloja la emoción recluida de quien ha perdido las facultades comunicativas. En la película El Profesional se reconoce con facilidad la relación sintomática. Alguien lleva la condición de vida solitaria, León. Está entrenado para actuar como criminal rentado. En un instante sintomático de comunicación, cultiva la instancia emocional del partener (Matilda), para darle un sentido a su vida.

Lo que hace especial a Matilda es que permite la inclusión de su inocencia en un episodio policial como motivo para una relación de compañerismo. Es partener desde la inocencia. Capaz de mantener conversaciones picarescas ante las que el protagonista solo tiene la opción de concebir como inocente. Provocaciones que se trasladan al espectador quizá para infundir la misma duda o solo para dotar al film de un atractivo mayor (Maldivia, 2011).

Al igual que Maia y Lucía, Matilda permite la salida del encierro sin sentido de alguien que ha extraviado la simbolización. Ambos adoptan una vida errante que se convierte, de pronto, en artística. Reconoce su extrañeza por sobre su inteligencia. Sentirse extraños permite renovar el juego. Entre tantos artistas que se amparan en el "síntoma" Jacques Lacan elige a Joyce y el acompañamiento de su hija. Es la prueba de que hay una posibilidad de anclaje poético en la extrañeza del partener. 


\section{Discusión. El síntoma como nuevo nomadismo}

Seguimos la reflexión de Néstor Rodríguez Pereira (2018, 133-136) al afirmar que se pone en tensión la capacidad normalizadora del psicoanálisis en términos de producir sujetos adaptados y adecuados a sus contextos. Hay que poner el énfasis en la posibilidad que ofrece para promover cambios en los mismos a partir de las propias demandas de quienes consultan. Uno de los descentramientos propuestos por el psicoanálisis tiene que ver con cómo se ha entendido tanto la sexualidad, como el amor, como el fenómeno nómade.

El paseante nómade está comprometido con su propia otredad. Si lo que se busca es conferir coherencia a la propia sociedad esa otredad es una "anomalía". Pero si se desea atender a discursos que van más allá de particularismos circunscritos, la coherencia no elude la diversidad sino que la asume. El viaje interior, el paseo dentro de sí mismo, permite un enlace con lo real en los casos vistos. En este artículo hemos expuesto cómo se manifiesta a modo de partener, el desplazamiento con alguien que nos sirve de guía para no caer en la ausencia pura.

Una cierta configuración persecutoria no deja de organizar el exilio de Joyce con relación a Irlanda, su tierra natal, pues el escritor, basándose en algunos acontecimientos relacionados con él o con su obra, mantenía firmemente la creencia de que era persona no querida en su propio país. No obstante llevaba a su Irlanda consigo como sostiene Jorge Luis Borges en una conferencia sobre Joyce dictada en la Universidad de La Plata en 1960, (2018). Es decir, que para que él escribiera esos dos libros tan irlandeses, el Ulises y el Finnegans Wake, fue necesaria la nostalgia, fue necesario el estímulo y el acicate, y esto Joyce lo supo. La otredad de Joyce es la cordura a la que no puede volver. Pasea por ciudades europeas. No es un paseo feliz ni tranquilo.

Los nuevos paseantes renuncian a sus hogares fijos. No vuelven a tener domicilio. Salen en busca de un hogar. Generan recursos suficientes como para vivir en un automóvil y trabajar en cualquier oficio. El "síntoma" se vuelve como desapegue del destino hogareño. Una búsqueda de sí mismo dentro del reverso de otredad. El nuevo nomadismo que cada uno de nosotros transporta proviene de la propia inocencia a la que nos ha arrojado la historia personal de cada uno. Una vida que se aleja de cálculos de aprovechamiento. 


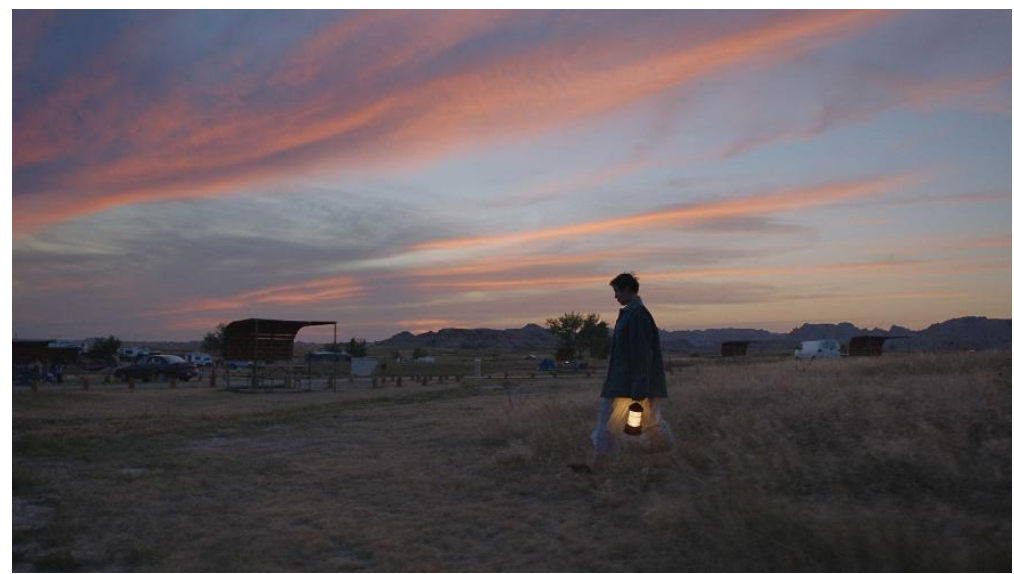

Fern cruza el acampe durante el atardecer. nomadland-recensione-venezia-4.jpg $\underline{(3840 \times 2160)}$ (leganerd.com)

La aproximación a la belleza del nomadismo no se limita a la naturaleza pletórica de los paisajes naturales, sino a la que se esconde en los pueblos deshabitados, los asentamientos de los actuales nómadas o las interminables carreteras rodeadas de desierto. El reciente film Nomadland se basa en un ensayo de la periodista norteamericana Jessica Bruder sobre jubilados nómadas en busca de trabajos itinerantes de baja remuneración. La autora celebra la capacidad de recuperación y creatividad de estos nuevos nómades, que han renunciado al arraigo ordinario. A través de su libro, y de su fiel interpretación en la pantalla, se capta un profundo respeto por esta forma de vida como una crítica ardiente de la sociedad que ha dejado a muchas de estas personas sin otra opción. Por cada gran paisaje filmado en la película, hay una escena de hormigas en camionetas o defecando en cubos, trabajos agotadores en fábricas o trabajos de limpieza de baños. Es un juego de supervivencia, no una aventura de año sabático.

\section{Referencias bibliográficas}

ALLIGOOD, M. y MARRIMER TOMEY, A. (2011). Modelos y Teorías en Enfermería. 7ma edición. España: ELSEVIER. Título original: Nursing theorists and their work.

BAUDELAIRE, C. (1964). "El pintor de la vida moderna". Nueva York: Da Capo Press, 1964. Publicado en Le Figaro (1863).

BORGES, J.L. (2018). Conferencia sobre James Joyce. Universidad Nacional de La Plata, 1960. Variaciones Borges, 2018, no 45, p. 207-220. https://www.jstor.org/ stable/26476379

BRUDER, J. (2017). Nomadland: Surviving America in the Twenty-First Century. New York, NY, W. W. Norton \& Company, 2017.

CANCLINI, A. (2009). El Fueguino. La cautivante historia de Jemmy Button (1ºd.). Buenos Aires, Argentina: Ediciones Monte Olivia. 
CANNATARO, M. (2021). "Buscan a Maia, nena de 7 años desaparecida". Actualizado a: 17 de marzo de 2021 09:40 https://argentina.as.com/argentina/2021/03/17/ actualidad/1615982567 436401.html

CHAPPET, M-C. (2021). Nomadland: Jessica Bruder details the gritty true story behind the Oscar-winning film. https://www.harpersbazaar.com/uk/culture/entertainment/ a36350360/jessica-bruder-nomadland-true-story/

FOUCAULT, M. (1974-1975). Les Anormaux. En Annuaire du Collège de France, 76 année. Histoire des systèmes de pensèe, années 1974-1975. 1975, pp. 335-339. Reeditado en Dits et Écrits, ob. cit., vol. 2, núm. 165, pp.822-828.

FOUCAULT, M. (1991). Las desviaciones religiosas y el saber médico. En la vida de los hombres infames. Altamira, 1991. La Plata.

GUILLEBAUD, J-C. (2000). La tiranía del placer. Barcelona: Andrés Bello.

HUTCHINS, P. (1957). James Joyce's world. London: Methuen and Co. Ltd, 1957. https://doi.org/10.4324/9781315624884

LACAN, Jacques Seminario 23 o Libro XXIII El sinthome. Editorial Paidós o actualmente Paidós-Planeta Libros.

LAIA, S. (2003). La locura de Joyce. Virtualia. Revista digital de la Escuela de la Orientación Lacaniana, 2003, vol. 8.

LAURENT, E. (2019). La Época del Sinthome 2019-11-27- LA ÉPOCA DEL SINTHOME. https://psicoanalisislacaniano.com/2019/11/27/epocadelsinthome-ericlaurent20191117/\# ftn1

LOMBROSO, C. (1895). L'homme criminel, étude anthropologique et médico-légale. Mencionado por GUILLEBAUD, Jean-Claude. La tiranía del placer. Barcelona: Andrés Bello, 2000. 427 p.

MALDIVIA, B. (2011). Críticas a la carta: 'El profesional (Léon)'. https://www.espinof.com/criticas/criticas-a-la-carta-el-profesional-leon

NICHOLS, P. (2003). La sombra de Darwin. Buenos Aires, Emecé.

SHAYA, G. S. (2004). The American Historical Review, Volumen 109, Número 1, febrero de 2004, páginas 41-77, https://doi.org/10.1086/ahr/109.1.41

* Aldo Enrici: Doctor en filosofía por la Universidad Autónoma de Madrid. Estancia Posdoctoral en Reims, Francia. Profesor titular ordinario de Estética en la Universidad Nacional de la Patagonia Austral (UNPA). Investigador categoría 1 del Programa Nacional De Incentivos. Extensionista categoría A. Director de becarios doctorales y posdoctorales de Conicet $y$ de universidades argentinas $y$ latinoamericanas. [E-mail: aenrici@uarg.unpa.edu.ar]

** Luciana Mansilla: Estudiante de turismo en la Universidad Nacional de la Patagonia Austral (UNPA). [E-mail: belen22lu@gmail.com] 